\title{
Are Impairments in Theory of Mind Specific to Borderline
}

\section{Personality Disorder?}

\author{
Normann-Eide, Eivind ${ }^{\mathrm{a}, \mathrm{f}}$ \\ Antonsen, Bjørnar Torske ${ }^{\mathrm{a}, \mathrm{e}}$ \\ Kvarstein, Elfrida Hartveit ${ }^{\text {a,b }}$ \\ Pedersen, Geir ${ }^{\mathrm{a}, \mathrm{c}}$ \\ Vaskinn, Anja ${ }^{c}$ \\ Wilberg, Theresa ${ }^{b, d}$
}

\section{Affiliations}

${ }^{\text {a }}$ Section for Personality Psychiatry, Clinic for Mental Health and Addiction, Oslo University Hospital, Oslo, Norway

${ }^{\mathrm{b}}$ Institute for Clinical Medicine, University of Oslo, Oslo, Norway

${ }^{c}$ NORMENT KG Jebsen Centre for Psychosis Research, Oslo University Hospital \& Institute of Clinical Medicine, University of Oslo, Norway

${ }^{\mathrm{d}}$ Department of Research and Development, Clinic for Mental Health and Addiction, Oslo University Hospital, Oslo, Norway

${ }^{\mathrm{e}}$ Lovisenberg Diaconal Hospital, Oslo, Norway

${ }^{\mathrm{f}}$ Research Department, Division of Mental Health and Addiction, Vestfold Hospital Trust, Tonsberg, Norway

Keywords: Borderline personality disorder, Social cognition, Theory of Mind, Hypermentalization, Interpersonal problems 


\begin{abstract}
Impaired social cognition (understanding feelings, intentions, and thoughts of others) is an assumed feature of borderline personality disorder (BPD). Yet, no studies have compared social cognitive abilities of patients with BPD, other personality disorders (OPD) and healthy controls (HC), or investigated the relationship between social cognition and overall severity of psychopathology and interpersonal problems. These were the aims of the present study.
\end{abstract}

In this study, theory of mind (ToM) was investigated by the video-based measure Movie for the Assessment of Social Cognition (MASC) applied in a clinical sample of men and women with BPD (n=53), OPD (n=34) and HC (n=71). BPD patients had greater comorbidity (other PD criteria, symptom disorders and symptom distress) than the OPD and HC groups. Other PD criteria and symptom distress were considered indicators of further severity. Statistics included multivariate analyses.

No differences were found between the three groups in overall social cognitive abilities. The BPD group was, however, characterized by more excessive ToM (interpreted as hypermentalization). Yet, when differentiating between BPD and further severity indicators, excessive ToM was not specifically associated with a BPD diagnosis per se. Finally, there was a moderate association between excessive ToM and interpersonal problems in the BPD group, whereas no such association was found in the OPD group.

This study suggests that BPD patients tendency to hypermentalize when they misinterpret social information is related to the severity of their psychopathology 


\section{Introduction}

Borderline personality disorder (BPD) is a severe psychiatric condition, characterized by marked disturbances in affect regulation, impulsivity, and interpersonal problems (Lieb et al, 2004; Gunderson et al, 2009). The disorder is prevalent in clinical populations (Zimmerman et al, 2005) and associated with severe symptom distress and poor psychosocial functioning (Skodol et al, 2002; Bly et al, 2012). Social cognition refers to the natural human capacity to perceive, interpret, and process all information related to a person's social environment and relationships (Sharp et al, 2012), and aberrancies in social cognition has increasingly been recognized as a central dysfunction in BPD (Sharp et al, 2013; Roepke et al, 2013; Fonagy et al, 2015; Vaskinn et al, 2015).

The social-cognitive construct Theory of mind (ToM) (Premack \& Woodruff, 1978) is encompassed within the concept of social cognition (Pinkham, 2014). It is defined as the capacity to interpret the behavior of others within a mentalistic framework - i.e. an individual's ability to perceive desires, feelings, thoughts, and beliefs in others and employ this ability to interpret, anticipate, and influence others' behavior (Sharp \& Vanwoerden, 2014). Hence, whereas the conceptual sibling mentalization, refers to the capacity to make sense of both one's own and other people's minds, ToM refers only to the understanding of mental states in others (Allen et al, 2008; Choi-Kain \& Gunderson, 2008; Kim, 2015). As misinterpretations of other people's mental state - e.g. experiencing another person as having threatening or repellent intentions - might represent situational cues for strong negative affects and interpersonal problems found in patients with BPD, ToM dysfunction is assumed to be a key factor for understanding their affective disturbances and interpersonal problems (Roepke et al, 2013; Vaskinn et al, 2015).

However, regarding measurements of social cognition and ToM, and the relationships with BPD psychopathology, evidence is scarce. As summarized by Roepke and colleges 
(2013), results from studies of social cognition in patients with BPD have been inconsistent, and an important limitation of prior studies is a lack of ecological validity. Previous studies are mostly based on still-face experiments (Baron-Cohen et al, 2001), or static or morphing pictures of facial affects (Domes et al, 2009). Accordingly, a major challenge in the study of social cognition has been the development of tests that are sufficiently close to real life interpersonal experiences and sensitive enough to capture the deviant relatedness expected in individuals with BPD (Preißler et al, 2010).

One test which provides a complex and ecologically more valid approach is the "Movie for the Assessment of Social Cognition" (MASC) (Dziobek et al, 2006). The MASC has been proposed to be a good candidate for studies that aim to increase the knowledge of social cognition in individuals with BPD pathology (Roepke et al, 2013). In this test the responses are classified as either a correct response or one of three alternative incorrect responses ("no ToM"; lack of mental state concept, "insufficient ToM"; reduced mental state reasoning, and "excessive ToM", over-interpretative mental state reasoning). Compared to former assessment methods, the MASC provides a more complex and nuanced measurement of social cognition, enabling a differentiation of the different incorrect responses (Dziobek et al, 2006; Roepke et al, 2013). Furthermore, the MASC test has been used in studies detecting mindreading problems in adolescents with borderline traits (Sharp et al, 2011; 2013), in adults with normal IQ (Dziobek et al, 2006), or with mental disorders, such as schizophrenia (Fretland et al, 2015; Vaskinn et al, 2015), bipolar disorder (Montag et al, 2010; Santos et al, 2017), social anxiety (Buhlmann et al, 2015) and in adult women with BPD (Preißler et al, 2010; Vaskinn et al, 2015).

Within the BPD research literature, excessive ToM is interpreted as hypermentalization (Sharp, et al 2011; Sharp et al, 2013; Bateman \& Fonagy, 2016), which is defined as social cognitive processes that involve making assumptions about other people's 
mental states that go far beyond observable phenomena (Sharp et al, 2013). A former study based on a subsample of the participants in the present study, compared MASC results for women with BPD, schizophrenia, and healthy female control participants (Vaskinn et al, 2015). One finding was that the BPD group was characterized by more hypermentalization errors. This result is comparable to earlier studies by Sharp and colleges who also demonstrated problems of hypermentalization among adolescents with BPD traits (Sharp et al, 2011). In that sample, improvement of hypermentalization problems was related to a reduction of BPD pathology (Sharp et al, 2013).

In a study comparing women with BPD (one third with comorbid PTSD) and nonclinical controls, Preißler and colleges (2010) reported that the BPD group had significantly more impairments in ToM as measured with MASC. No differences were found when using the less ecologically valid "Reading the mind in the eyes test" (Baron-Cohen et al, 2001). Moreover, clinical factors contributing to the impaired ToM in the BPD group were comorbid PTSD intrusive symptoms and a history of sexual assaults. These results suggest that ToM problems in individuals with BPD increase with more severe psychopathology.

Impairment in social cognition is not only a feature of BPD samples, but has also been demonstrated in samples with other personality disorders (PDs) (Herpertz \& Bertsch, 2014). Ritter and colleges (2011) reported a significantly impaired emotional empathy in patients with narcissistic PD (NPD) and patients with BPD, compared to healthy controls (HC). Moreover, in a comparison of patients with NPD, cluster C PDs and HC (Marlies et al, 2012) recognition of facial emotional expressions in others was impaired for NPD patients when measured with the self-reported "Interpersonal Reactivity Index" (Davis, 1983) and a facial emotion recognition task (Ekman and Friesen, 1976; Young et al., 2002). In that study, there was a significant negative association between accuracy in facial recognition and severity of symptom distress. Together, the findings of Preißler et al, and Marlies et al, suggest that not 
only specific PDs, but the overall severity of psychopathology associated with the disorder has an impact on ToM.

Moreover, in a study comparing metacognition in patients with BPD and other PDs (OPD), Semerari and colleges (2015) did not find differences between BPD and OPD patients in their ability to make reasonable hypotheses about other people's mental states (termed "Decentration"), when they controlled for symptomatic distress and severity of personality pathology. This finding lends further support to the notion that ToM problems could be associated with the severity of PD, rather than specific features of BPD pathology per se.

It is widely recognized that patients with BPD are characterized by an impaired ability to make appropriate assumptions about other people's mental states (New et al, 2012; Fonagy et al, 2015), and previous studies have found subtle impairments in social cognition related to BPD. However, the specificity of this relationship is not known, and it is currently not clear to what degree such dysfunction is related to the severity of psychopathology associated with BPD. Our former study (Vaskinn et al, 2015) compared ToM in female patients with BPD, schizophrenia, and HC, and was not designed to investigate these issues. The present study therefore aimed to compare ToM as assessed by MASC, in a mixed gender sample of patients with BPD, patients with OPDs and HC. We investigated 1) level of ToM dysfunction, 2) differences in error distributions, 3) the effect of more severe psychopathology, and 4) associations between particular ToM errors and interpersonal problems.

\section{Hypotheses:}

1. Individuals with BPD, OPD, and $\mathrm{HC}$ do not differ in number of correct MASC responses.

2. MASC error patterns differ according to PD pathology. More excessive ToM errors will be committed in the BPD group than in the OPD and HC groups. 
3. Excessive ToM is associated with the overall severity of psychopathology characterizing patients with BPD, indexed by total number of co-occurring PD criteria and global level of symptom distress.

4. Excessive ToM is positively associated with level of interpersonal problems.

\section{Methods}

\section{Settings}

The present study was conducted at the Section for Personality Psychiatry (SPP) and NORMENT KG Jebsen Centre for Psychosis Research at Oslo University Hospital in Norway. SPP is a tertiary level outpatient unit specialized in treatment of patients with PDs. Experienced clinicians at the SPP conducted and organized the clinical interviews and selfreport measures of the participants with PDs. HCs from the same geographical areas as the participants with PDs were recruited from the Thematically Organized Psychosis (TOP) Study at NORMENT. They were screened with an interview to capture symptoms of severe mental illness (Primary Care Evaluation of Mental disorders; PRIME-MD; Spitzer el al, 1994), and excluded if there were any signs of mental, somatic, or neurological disorder. Written informed consent was obtained from participants after complete description of the study. The study was approved by the Norwegian Data Inspectorate and the Regional Committee for Medical Research Ethics.

\section{Assessments}

\section{Theory of mind}

ToM was assessed with the video-based instrument MASC (Dziobek et al., 2006), a 15-min movie about four people coming together for a dinner party. The movie is paused 45 times, and the study participants are given questions concerning the characters' feelings, thoughts, 
and intentions (e.g. "what is Cliff feeling?", "what is Michael thinking?", and "why is Sandra asking this?"). At each pause, participants are provided with four response options: 1) an accurate response, suggesting intact ToM, 2) an excessive ToM response, 3) an insufficient ToM response, and 4) a no ToM response. Excessive ToM reflects assumptions about other people's mental states, beyond what most other observers would find reasonable. Insufficient ToM reflects mental state inferences that are not satisfactory. No ToM reflects non-mental state inferences, e.g. physical causation (Dziobek et al, 2006; Vaskinn et al., 2015).

\section{Diagnoses}

PD diagnoses were determined according to the $4^{\text {th }}$ edition of the Diagnostic and Statistical Manual of Mental Disorders (DSM) and the Structured Clinical Interview for DSM-IV Axis II Disorders (SCID-II) (First, 1994). Symptom disorder diagnoses were based on the Mini International Neuropsychiatric Interview (M.I.N.I: Sheehan \& Lecrubier, 1994).

\section{Psychosocial functioning}

Psychosocial functioning was evaluated by Global Assessment of Functioning (GAF: APA, 1994). GAF is an observer based single score ranging from 1-100, representing symptom severity and social and occupational impairment. In a study by Pedersen et al, (2007) reliability of GAF was found acceptable, with a generalizability coefficient of .84 , for relative decision and approximately .82 for absolute decisions. Conventional interpretations of severity indicated by GAF scores are: Mild (61-70); Moderate (51-60); and Severe (41-50) (APA, 1994).

\section{Symptom distress}


Symptom distress was assessed by the Revised Symptom Checklist-90 (SCL-90-R)

(Derogatis, 1982). The SCL-90-R is a self-report questionnaire rated on a five point Likert scale, from 0-4. It is designed to cover nine areas of symptom distress, and the mean score of all 90 items, the Global Severity Index (GSI) represents the overall level of distress. A higher score indicates more symptom distress.

\section{Interpersonal problems}

Interpersonal personal problems were assessed by the Circumplex of Interpersonal Problems (CIP) (Pedersen 2002). This self-report questionnaire is a 48-item version of the Inventory of Interpersonal Problems (Alden et al., 1990). It comprises eight subscales summarized in an Index of Interpersonal problems (IIP). The items are rated on a five-point Likert scale from 0 to 4; a higher score indicates more severe interpersonal problems.

\section{Personality functioning}

Severity Indices of Personality Problems (SIPP-118) is a self-report questionnaire for the assessment of core components of (mal) adaptive personality functioning that are believed to be changeable (Andrea et al., 2007; Verheul et al., 2008; Bastiaansen et al., 2013). The questionnaire consists of 118 items rated on a four point Likert scale (1-4) covering 16 facets representing core components of (mal) adaptive capacity, organized in five higher-order domains, Self-Control, Identity Integration, Relational Capacities, Social Concordance, and Responsibility (Verheul et al., 2008). Higher scores on the facets and domains of SIPP are presumed to indicate more adaptive and thus less pathological capacities. In the present study, Relational Capacities was applied as a measure of interpersonal functioning. The Norwegian translation of the SIPP-118 was adapted in accordance with the guidelines of Hambleton (2005) by a group of eight clinicians and researchers (Pedersen, Arnevik, Walderhaug, 
Hummelen \& Wilberg, 2017). Although the factor structure of SIPP-118 has not been fully settled (Bastiaansen et al., 2013, Pedersen et al., 2017), three studies have reported good psychometric properties of the 16 facets in adults and adolescents (Verheul et al., 2008; Feenstra et al., 2011), including cross-national consistency in adult PD populations (Arnevik et al., 2009).

\section{Participants}

The present study comprises 153 participants. Demographics and clinical characteristics of the sample are presented in Table 1. Participants with BPD $(n=53)$ and OPD $(n=34)$ were recruited from the SPP, whereas the healthy control participants $(\mathrm{HC}, \mathrm{n}=71)$ were recruited from TOP. There was a higher number of females in the BPD group compared to both the OPD group ( $p<.01)$ and the HC group ( $p<.001)$, and correspondingly in the OPD group compared to the HC group $(p<.05)$. Neither age nor gender were associated with any of the MASC variables.

\section{Statistical analysis}

All analyses were performed using SPSS statistics version 21 (IBM SPSS Statistics for Windows, Version 21.0. Armonk, NY: IBM Corp). The first research question, regarding group differences in MASC responses, was investigated using a univariate analysis of variance (ANOVA) with post hoc Scheffé comparisons. The MASC variable total correct responses, was entered as the dependent variable, and diagnostic group (BPD/OPD/HC) as independent variable. For our second research question a two-way mixed ANOVA was conducted with one within-subjects factor (the three MASC error scores: excessive ToM/insufficient ToM/no ToM) and one between-subjects factor (diagnostic group: $\mathrm{BPD} / \mathrm{OPD} / \mathrm{HC}$ ). A significant error $\mathrm{x}$ diagnostic group interaction indicates that error patterns 
depend on diagnostic group membership. Spearman`s rank order correlation was used to test the statistical relationships between the MASC variables and types of PD criteria and clusters. The third research question was investigated by an ANCOVA with excessive ToM entered as the dependent variable, BPD diagnosis as fixed factor and Other PD criteria and GSI as covariates. Finally, our last research question, regarding the relationship between MASC scores and interpersonal functioning as measured by CIP and the SIPP-118 domain Relational Capacities, was investigated using Spearman`s correlation.

\section{Results}

The ANOVA revealed that there was no significant group effect in overall social cognition.

Differences in MASC total correct responses between the BPD, OPD, and HC group were not statistically significant $\left[F(2)=0.28, p=0.76, \eta^{2}<0.01\right]$. In the two-way mixed ANOVA, the main between-subject effect of diagnostic group was not significant $[F(2)=0.31, p=0.74$, $\left.\eta^{2}<0.01\right]$. Mauchly's test indicated that the assumption of sphericity was violated $\left[\mathrm{x}^{2}\right.$ $(2)=62.4, p<0.001]$, and degrees of freedom was corrected using Greenhouse-Geisser estimates of sphericity. The main within-subjects effect or error type was significant $[F(1.5)=77.56, p<0.001, \eta=0.33]$, as well as the error type $\mathrm{x}$ diagnostic group interaction effect $[F(3)=28.28, p<0.01, \eta=0.05]$. See Table 2 and Figure 1 . These results suggest that the compared groups differ in error patterns. Excessive ToM errors were more frequent among BPD patients than OPD and HC.

The associations between excessive ToM and PD pathology are illustrated in Table 3. There were statistically significant positive correlations between excessive ToM and total number of PD criteria, excessive ToM and total number of PD criteria other than BPD, and excessive ToM and number of cluster $\mathrm{A}$ and cluster $\mathrm{C}$ criteria. The number of excessive ToM errors was not significantly associated with number of BPD criteria or cluster B criteria. 
As the BPD group was characterized by a significantly higher number of other PD criteria, symptom disorder diagnoses and a higher level of symptom distress, we investigated whether the excessive ToM problems were particularly related to BPD or to the overall severity of psychopathology characterizing the BPD group. We applied number of Other PD criteria and GSI as indices of severity of pathology and conducted an ANCOVA with excessive ToM as dependent variable and BPD as fixed factor. When Other PD criteria and GSI were entered as covariates, the association between excessive ToM and BPD was no longer statistically significant. Moreover, an interaction between BPD and Other PD criteria did not contribute significantly to explained variance in excessive ToM. To explore the significance of specific co-occurring PD traits we performed a series of ANCOVAs with excessive ToM as dependent variable and BPD, paranoid, histrionic, or obsessive PD criteria as covariates in separate analyses, as well as interactions between BPD and paranoid, histrionic, or obsessive-compulsive traits respectively. None of the covariates or interaction variables were statistically significant.

Finally, we investigated the relationship between MASC performance and interpersonal problems, for all PD patients as one group, and then separately for the two clinical groups. In the total PD group there were no significant correlations between excessive ToM and interpersonal problems. However, when we separated the BPD and OPD group, the results showed a significant positive correlation between excessive ToM and level of interpersonal problems in the BPD group, as measured by CIP $(\mathrm{r}=.29, p=.04)$. There were no significant relationships between excessive ToM in the BPD group and interpersonal problems as measured with SIPP-118, or between any of the variables in the OPD group. The other MASC variables were unrelated to interpersonal problems in both groups.

\section{Discussion}


The aim of the present study was to examine ToM in patients with BPD, compared to other PDs and HCs. We found no differences in overall ToM between the three groups, indicating that patients with BPD and OPDs are not impaired compared to HCs when it comes to ToM. This is in line with the conclusions of the review by Roepke and colleges (2013) who emphasized that several studies have failed to demonstrate impairments in social cognition in patients with BPD. However, as previously pointed out by Sharp and colleges (2016), it has been difficult to demonstrate empirically the well-known and clinically visible problems involved in the interpretation of other people's minds that are associated with BPD psychopathology.

The second finding of the present study was that patients with BPD displayed different error distributions. When they made mistakes, they had more hypermentalization errors compared to individuals with OPDs or to HCs, which suggests that ToM difficulties in BPD patients are characterized by a tendency to add more into the interpretation of other people's mind, than most other would find reasonable. This result is in line with previous studies on adolescents with BPD pathology (Sharp et al, 2011; Sharp et al, 2013), as well as adult females with BPD (Vaskinn et al, 2015).

In a recent study, Sharp and colleges (2016) found that more severe borderline pathology in adolescents was associated with more hypermentalization errors, and that these errors mediated a link between attachment insecurity and BPD psychopathology. In line with their results, Fonagy \& Bateman (2016) argue that hypermentalization is uniquely linked to BPD features. In contrast to this assumption, the present findings suggest that the increased hypermentalization found in patients with BPD might rather be influenced by the severity of symptom distress and extent of PD pathology in general, and not merely the BPD features. In our study, there was a statistically significant association between hypermentalization and the total number of PD traits other than BPD. The association between BPD and 
hypermentalization was no longer significant when we controlled for symptom distress and other PD criteria. Accordingly, the present results support a dimensional approach to the understanding of PD pathology, as some of the ToM strategies that characterize these patients seem more linked to the severity of psychopathology than the PD category in itself.

This is in line with a study by Semerari and colleagues (2015) which investigated metacognitive dysfunctions in patients with BPD. In that study impaired ability to reflect upon other's intentions, thoughts, and desires ("Decentration"), was not only found among patients with BPD, but in a group of patients with other PDs as well. When they controlled statistically for severity in terms of total number of PD criteria and symptom distress, "Decentration" was associated with severity of psychopathology, and not specifically with BPD pathology. The term "Decentration" corresponds to the definition of ToM, and it might be, as previously emphasized by Herpertz and Bertsch (2014), that social cognitive problems characterize individuals with PDs per se.

There has been an ongoing debate as to what degree PD pathology is accounted for by a general dimensional factor and/or more specific factors (Morey \& Bender, 2014). In a large clinical sample, Sharp and colleges (2015) found support for a general factor that captured common variance across different PDs, and six specific factors capturing unique sources of variance. In that study, the BPD items, measured with the SCID-II interview, loaded most strongly on the general factor, suggesting that the BPD criteria represent a prototype for PD. Hence, hypermentalization might be associated with BPD insofar as BPD could be the most prototypical of personality disorders. On the other hand, the present results also suggest that particular types of ToM dysfunctions, rather than being linked to specific PD types, could be an aspect of a general PD factor, lying at the core of different manifestations of personality pathology. Moreover, an inclination for hypermentalization may reflect a general severity of personality pathology, here represented by a continuum of symptom distress and co-occurring 
PD traits. This particular kind of ToM dysfunction makes a person susceptible to distortions in his or her interpretation of others mind, e.g., patients with severe PDs tend to have negative perceptions of others and to be suspicious of others intentions (Dimaggio \& Brüne, 2016; Herpertz \& Bertsch, 2014). Such susceptibility may contribute to the interpersonal problems commonly found in these patients. There is, however, a need for more studies that investigate the clinical implications of different types of ToM errors in patients with severe PDs.

In the present study there was an association between hypermentalizing errors and interpersonal problems in the BPD group, whereas there was no such relationship in the OPD group. However, in these analyses we did not take into account the clinical severity of pathology in the two groups. In BPD the importance of interpersonal disturbances have been reported in several studies (i.e. Sanislow et al, 2002; Skodol et al, 2005; Gunderson \& LyonsRuth, 2008; Drapeau et al, 2012; Lazarus et al, 2014), and disturbed interpersonal function, together with affective instability and impulsivity, is suggested to represent a major borderline phenotype (Gunderson, 2007). Moreover, as emphasized by Clarkin and colleges (2007), intense emotions are activated together with corresponding cognitive systems. E.g. an angry outburst might be legitimized by hypermentalization errors, in terms of a (for the patient) convincing interpretation of the other as hostile or not affectionate, justifying the intense emotion. Interestingly, in a study of women with BPD, Sadikaj and colleges (2010) found that dysregulation of negative affects was triggered by interpretations of being rejected or abandoned in close relationships.

The relationship found in the present study, between hypermentalization and relational problems as measured with CIP, support these previous findings. However, the association was not strong and hypermentalization was unrelated to the SIPP-118 domain Relational Capacities. It might be that these self-report instruments capture different phenomena, e.g. that CIP expresses interpersonal problems more related to hypermentalization. Also, the factor 
structure of the SIPP-118 is not fully clarified and future studies should investigate these relationships on the facet level of SIPP-118 (Pedersen et al, 2017). Moreover, it is hypothesized that mentalizing breakdowns occur particularly during high emotional arousal in close relationships (Bateman \& Fonagy, 2016). An association between hypermentalization and interpersonal difficulties might therefore be more profound when engaging personally in real life interpersonal situations. Despite greater ecological validity than former tests, the MASC is probably not able to create situations with emotional involvement comparable to distressing, real-life relational situations.

A strength of the present study was the inclusion of both OPD and $\mathrm{HC}$ as comparison groups, as well as both male and female participants. The BPD and OPD groups comprised well-diagnosed patients with moderate to severe dysfunction. However, some limitations should be considered. First, the number of participants in the BPD and the OPD groups were relatively small, and the diagnostic distribution in the OPD group was mainly avoidant PD and PD NOS. Second, the HC participants were not screened for personality pathology or interpersonal problems in particular, and we cannot rule out the possibility that some might have maladaptive personality traits. However, as they were screened to capture symptoms of severe mental illness in general, and excluded if there was any information on mental, neurological, or somatic disorder, significant problems related to PD pathology seem unlikely. Third, even though the participants in the BPD and OPD groups had co-occurring traits from different PD types, the results may not generalize to a broader range of PDs. Finally, the OPD group was characterized by less symptomatic distress and general PD pathology compared to the BPD group. Although these differences were taken into account in the statistical analyses, larger PD groups with comparable levels of psychopathology would have strengthened the validity of conclusions. 
In summary, the present study did not reveal any group differences in overall ToM dysfunction. Patients with BPD may be prone to hypermentalization, but this seems to be related to the overall extent and severity of psychopathology rather than merely BPD. Moreover, even though impairment in ToM is subtle and hard to measure, the MASC test indicates characteristic problems among PD patients. Although hypermentalization has been associated with BPD pathology, future studies should specifically address its association with other PDs and the impact of overall severity of personality pathology. More knowledge of cognitive processes involved in the understanding of others mind and behavior in patients with PDs is clinically relevant, and may contribute to the understanding of their interpersonal difficulties as well as patient-therapist interactions and processes during treatment. 


\section{References:}

Alden, L. E., Wiggins, J. S., \& Pincus, A. L. (1990) Construction of circumplex scales for the inventory of interpersonal problems. Journal of Personality Assessment, 55, 521-536.

Allen, J. G., Fonagy, P., \& Bateman, A. W. (2008). Mentalizing in Clinical Practice. American Psychiatric Publishing. Washington DC.

Andrea, H., Verheul, R., Berghout, C. C., Dolan, C., van der Kroft, P. J. A., Bateman, A. W., \& Fonagy, P. (2007). Measuring the core components of maladaptive personality: Severity indices of personality problems (SIPP-118). Report of the Viersprong Institute for Studies on Personality Disorders (VISPD) in cooperation with the Department of Medical Psychology \& Psychotherapy, Erasmus University Rotterdam, The Netherlands. Available: http://hdl.handle.net/1765/10066.

American Psychiatric Association (1994). Diagnostic and Statistical Manual of Mental Disorders. Fourth edition. Washington DC.

Arnevik, E., Wilberg, T., Monsen, J. T., Andrea, H., \& Karterud, S. (2009). A cross-national validity study of the Severity Indices of Personality Problems (SIPP-118). Personality and Mental Health, 3, 41-55.

Baron-Cohen, S., Wheelwright, S., Hill, J., Raste, Y., \& Plumb, I. (2001). The 'Reading the Mind in the Eyes' test revised version: a study with normal adults, and adults with Asperger's syndrome or high-functioning autism. Journal of Child Psychology and Psychiatry, 42, 241251. doi: $10.1111 / 1469-7610.00715$ 
Bateman, A.W., \& Fonagy, P. (2016). Mentalization-Based Treatment for Personality Disorders. A Practical Guide. Oxford University Press.

Bastiaansen, L., De Fruyt, F., Rossi, G., Schotte, C., \& Hofmans, J. (2013). Personality disorder dysfunction versus traits: Structural and conceptual issues. Personality DisordersTheory Research and Treatment, 4, 293-303.

Buhlmann, U., Wacker, R., \& Dziobek, I. (2015). Inferring other people's states of mind: Comparison across social anxiety, body dysmorphic, and obsessive-compulsive disorders. Journal of Anxiety Disorders, 34, 107-113.

Bly, E. M., Wright, A. J., \& Tuber, S. B. (2012). Unemployed in New York: The impact of mentalization and Axis II psychopathology on job outcome. Bulletin of the Menninger Clinic, 76, 101-129.

Choi-Kain, L. W., \& Gunderson, J. G. (2008). Mentalization: Ontogeny, assessment, and application in the treatment of borderline personality disorder. The American Journal of Psychiatry, 165, 1127-1135.

Clarkin, J. F., Lenzenweger, M. F., Yeomans, F., Levy, K. N., \& Kernberg, O. (2007). An Object Relations Model of Borderline Pathology. Journal of Personality Disorders, 21, 474499. 
Davis, M. H. (1983). Measuring individual differences in empathy: evidence for a multidimensional approach. Journal of Personality and Social Psychology, 44, 113-126.

Derogatis, L. R. (1983). SCL-90-R manual: administration, scoring \& procedures. Manual II. Baltimore, MD: Clinical Psychometric Research.

Dimaggio, G., \& Brüne, M. (2016). Dysfunctional understanding of mental states in personality disorders: What is the evidence? Comprehensive Psychiatry, 64, 1-3.

Domes, G., Schulze, L., \& Herpertz, S. (2009). Emotion Recognition in Borderline Personality Disorder - A Review. Journal of Personality Disorders, 23, 6-19.

Drapeau, M., Perry, J. C., \& Körner, A. (2012). Interpersonal Patterns in Borderline Personality Disorder. Journal of Personality Disorders, 26, 583-592.

Dziobek, I., Fleck, S., Kalbe, E., Rogers, K., Hassenstab, J., Brand, M., Kessler, J., Woike, J. K., et al. (2006). Introducing MASC: A movie for the assessment of social cognition. Journal of Autism and Developmental Disorders, 36, 623-636.

Ekman, P., \& Friesen, W. V. (1976). Pictures of Facial Affect. Consulting Psychologists Press. Palo Alto.

Feenstra, D. J., Hutsebaut, J., Verheul, R., \& Busschbach, J. J. V. (2011). Severity Indices of Personality Problems (SIPP-118) in Adolescents: Reliability and Validity. Psychological Assessment, 23, 646-655. 
First, M.B. (1994). Structural clinical interview for DSM-IV Axis II personality disorders (version 2.0). New York: New York State Psychiatric Institute.

Fonagy, P., Luyten, P., \& Bateman, A. W. (2015). Translation: Mentalizing as Treatment Target in Borderline Personality Disorder. Personality Disorders-Theory Research and Treatment, 6, 380-392.

Fonagy, P., \& Bateman, A. W. (2016). Adversity, attachment, and mentalizing. Comprehensive Psychiatry, 64, 59-66.

Fretland, R. A., Andersson, S., Sundet, K., Andreassen, O. A., Melle, I., \& Vaskinn, A. (2015). Theory of mind in schizophrenia: error types and associations with symptoms. Schizophrenia Research, 162, 42-46. doi: 10.1016/j.schres.2015.01.024.

Gunderson, J. G., \& Lyons-Ruth, K. (2008). BPD’s Interpersonal Hypersensitivity Phenotype: A Gene-Environmental-Developmental Model. Journal of Personality Disorders, 22, 22-41.

Gunderson, J. G. (2007). Disturbed Relationships as a Phenotype for Borderline Personality Disorder. The American Journal of Psychiatry, 164, 1637-1640.

Gunderson, J. G. (2009). Borderline Personality Disorder: Ontogeny of a Diagnosis. The American Journal of Psychiatry, 166, 530-539. 
Hambleton, R. K. (2005). Issues, designs and technical guidelines for adapting test into multiple languages and cultures. In R. K. Hambleton, P. F. Merenda, \& S. D. Spielberger (Eds.), Adapting educational and psychological test for cross-cultural assessment (pp. 3-38). Mahwah, NJ: Lawrence Erlbaum Associates.

Herpertz, S.C., \& Bertsch, K. (2014). The social-cognitive basis of personality disorders. Current Opinion in Psychiatry, 27, 73-77.

Kim, S. (2015). The Mind in the Making: Developmental and Neurobiological Origins of Mentalizing. Personality Disorders-Theory Research and Treatment, 6, 356-365.

Lazarus, S. A., Cheavens, J. S., Festa, F., \& Rosenthal, M. Z. (2014). Interpersonal functioning in borderline personality disorder: A systematic review of behavioral and laboratory-based assessments. Clinical Psychology Review, 34, 193-205.

Lieb, K., Zanarini, M. C., Schmahl, C., Linehan, M. M., \& Bohus, M. (2004). Borderline Personality Disorder. Lancet, 364, 453-461.

Marlies, M. A. E., Deen, M. L., \& Franken, I. H. A. (2012). Disturbed emotion recognition in patients with narcissistic personality disorder. Psychiatry Research, 198, 269-273.

Montag, C., Ehrlich, A., Neuhaus, K., Dziobek, I., Heekeren, H.R., Heinz, A., \& Gallinat, J. (2010). Theory of mind impairments in euthymic bipolar patients. Journal of Affective Disorders, 123, 264-269. 
Morey, L. C., \& Bender, D. S. (2014). Articulating a core dimension of personality pathology. In J. M. Oldham, A. E. Skodol, \& D. S. Bender (red.), Textbook of personality disorders, 2nd ed. Arlington, VA: APA.

New, A. S., Rot, M. A. H., Ripoll, L. H., Perez-Rodriguez, M. M., Lazarus, S., Zipursky, E., Wienstein, S. R., Koenigsberg, H. W. et al. (2012). Empathy and Alexithymia in Borderline Personality Disorder: Clinical and Laboratory Measures. Journal of Personality Disorders, 26, 660-675.

Pedersen, G.A. (2002). Revised Norwegian version of inventory of interpersonal problemsCircumplex (IIP-C). Tidsskrift for Norsk Psykologforening, 39, 25-34.

Pedersen, G., Hagtvet, K.A., \& Karterud, S. (2007). Generalizability studies of the Global assessment of functioning (GAF) - Split version. Comprehensive Psychiatry, 48, 88-94.

Pedersen, G., Arnevik, E., Walderhaug, E., Hummelen, B., \& Wilberg, T. (2017).

Psychometric Properties of the Severity Indices of Personality Problems (SIPP) in a Norwegian Community Sample and in Clinical Samples of Patients with and without Personality Disorders. European Journal of Psychological Assessment. doi: 10.1027/1015$5759 / \mathrm{a} 000436$.

Pinkham, A. E. (2014). Social cognition in Schizophrenia. Journal of Clinical Psychiatry, 75, 14-19. 
Premack, D., \& Woodruff, G. (1978). Does the chimpanzee have a 'theory of mind'? Behavioral and Brain Sciences, 1, 515-526.

Preißler, S., Dziobek, I., Ritter, K., Heekeren, H. R., \& Roepke, S. (2010). Social cognition in borderline personality disorder: evidence for disturbed recognition of the emotions, thoughts, and intentions of others. Frontiers in Behavioral Neuroscience. 4:182. doi:

10.3389/fnbeh.2010.0018

Ritter, K., Dziobek, I., Preissler, S., Ruter, A., Vater, A., Fydrich, T., Lammers, C. H., Heekeren, H. R., \& Roepke, S. (2011). Lack of empathy in patients with narcissistic personality disorder. Psychiatry Research, 187, 241-247.

Roepke, S., Vater, A., Preißler, S., Heekeren, H. R., \& Dziobek, I. (2013). Social cognition in borderline personality disorder. Frontiers in Neuroscience. 6:195. doi: 10.3389/fnins.2012.00195

Sadikaj, G., Russel, J. J., Moskowiz, D. S., \& Paris, J. (2010). Affect Dysregulation in Individuals with Borderline Personality Disorder: Persistence and Interpersonal Triggers. Journal of Personality Assessment, 92, 490-500.

Sanislow, C. A., Grilo, C. M., Morey, L. C., Bender, D. S., Skodol, A. E., Gunderson, J. G., Shea, M. T., Stout, R. L., et al. (2002). Confirmatory Factor Analysis of DSM-IV Criteria for Borderline Personality Disorder: Findings From the Collaboratory Longitudinal Personality Disorder Study. The American Journal of Psychiatry, 159, 284-290. 
Santos, J. M., Pousa, E., Soto, E., Comes, A., Roura P., Arrufat, F. X., \& Obiols, J. E. (2017). Theory of Mind in Euthymic Bipolar Patients and First-Degree Relatives. Journal of Nervous and Mental Disease, 205, 207-212.

Semerari, A., Colle, L., Pellecchia, G., Carcione, A., Conti, L., Fiore, D., Moroni, F., Nicolò, G., et al. (2015). Personality Disorders and Mindreading. Specific Impairments in Patients with Borderline Personality Disorder Compared to Other PDs. Journal of Nervous and Mental Disease, 203, 626-631.

Sharp, C., Ha, C., Carbone, C., Kim, S., Perry, K., Williams, L., \& Fonagy, P. (2013). Hypermentalizing in adolescent inpatients: treatment effects and association with borderline traits. Journal of Personality Disorders, 27, 3-18.

Sharp, C., Pane, H., Ha, C., Venta, A., Patel, A.B., Sturek, J., \& Fonagy, P. (2011). Theory of mind and emotion regulation difficulties in adolescents with borderline traits. Journal of the American Academy of Child \& Adolescent Psychiatry, 50, 563-573. doi:0.1016/j.jaac.2011.01.017

Sharp, C., Fonagy, P., \& Allen, J. G. (2012). Posttraumatic Stress Disorder: A SocialCognitive Perspective. Clinical Psychology-Science and Practice, 19, 229-240.

Sharp, C., \& Vanwoerden, S. (2014). Social Cognition: Empirical Contribution. The Developmental Building Blocks of Psychopathic Traits: Revisiting the Role of Theory of Mind. Journal of Personality Disorders, 28, 78-95. 
Sharp, C., Wright, Aidan G. C., Fowler, J. C., Frueh, B. C., Allen, J. G., Oldham, J., \& Clark, L. A. (2015). The Structure of Personality Pathology: Both General ('g') and Specific ('s') Factors? Journal of Abnormal Psychology, 124, 387-398.

Sharp, C., Venta, A., Vanwoerden, S., Schramm, A., Ha, C., Newlin, E., Reddy, R., \& Fonagy, P. (2016). First empirical evaluation of the link between attachment, social cognition and borderline features in adolescents. Comprehensive Psychiatry, 64, 4-11.

Sheehan, D. V. \& Lecrubier, Y. (1994). Mini-international neuro-psychiatry interview (M.I.N.I.). Tampa (Fla): University of South Florida Institute for Research in Psychiatry/INSERM-Hospital de la Salpétrière.

Skodol, A. E., Gunderson, J. G., Pfohl, B., Widiger, T. A., Livesley, W. J., \& Siever, L. J. (2002). The Borderline Diagnosis I: Psychopathology, Comorbidity, and Personality Structure. Biological Psychiatry, 51, 936-950.

Skodol, A. E., Gunderson, J. G., Shea, M. T., McGlashan, T. H., Morey, L. C., Sanislow, C. A., Bender, D. S., Grilo. C. M., et al. (2005). The Collaborative Longitudinal Personality Disorders Study (CLPS): Overview and Implications. Journal of Personality Disorders, 19, 487-504.

Spitzer, R.L., Williams, J.B., Kroenke, K., Linzer, M., deGruy, F.V. $3^{\text {rd }}$, Hahn, S.R., et al (1994). Utility of a new procedure for diagnosing mental disorders in primary care. The PRIME-MD 1000 study. JAMA, 272, 1749-1756. doi: $\underline{10.1001 / j a m a .1994 .03520220043029}$

Verheul, R., Andrea, H., Berghout, C. C., Dolan, C., van Busschbach, J. J., van der Kroft, P. J. A., Bateman, A. W., \& Fonagy, P. (2008). Severity indices of personality problems (SIPP- 
118): Development, factor structure, reliability, and validity. Psychological Assessment, 20, $23-34$.

Vaskinn, A., Antonsen, B. T., Fretland R. A., Dziobek, I., Sundet, K., \& Wilberg, T. (2015). Theory of mind in women with borderline personality disorder or schizophrenia: differences in overall ability and error patterns. Frontiers in Psychology, 6:1239. doi:10.3389/fpsyg.201501239.

Young, A. W., Perrett, D. I., Calder, A. J., Sprengelmeyer, R., \& Ekman, P. (2002). Facial Expressions of Emotion: Stimuli and Tests (FEEST). Thames Valley Test Company, Bury St. Edmunds, England. Computer Software.

Zimmerman, M., Rothschild, L., \& Chelminski, I. (2005) The prevalence of DSM-IV personality disorders in psychiatric outpatients. The American Journal of Psychiatry, 162, 1911-1918. 
Table 1: Socio-demographic and Clinical Characteristics

Age, mean (SD)

Females, \%

Education (years), mean (SD)

Self-harming behavior last year, \%**

Suicide attempts last year, \%

Number of BPD traits, mean (SD)***

No. of PDs $* * *$

No. PD criteria, mean (SD) $* * *$

Paranoid PD, \%***

Avoidant PD, \%**

Antisocial PD, \%

Obsessive-compulsive PD, \%*

Dependent PD, \%

Narcissistic PD, \%

PD NOS, $\% * * *$

No. symptom disorders, mean (SD) *

Symptom distress (GSI), mean (SD)**

Global Assessment Functional (GAF) Scale***

Interpersonal Problems (CIP), mean (SD)

Relational Capacities (SIPP-118)
$\operatorname{BPD}(\mathrm{n}=53) \quad$ OPD $(\mathrm{n}=34) \quad \mathrm{HC}(\mathrm{n}=71)$

$26.5(4.6) \quad 27.2(5.8) \quad 29.3(7.7)$

90.1

61.8

40.8

$14.3(2.7) \quad 14.7(2.8)$

90.5

67.6

26.5

9.7

$6.5(1.2) \quad 1.8(1.6)$

$1.7(0.9) \quad 1.0(0.2)$

$17.1(6.7) \quad 10.0(4.1)$

$24.5 \quad 0$

$20.8 \quad 50$

$3.7 \quad 2.9$

$13.2 \quad 0$

$7.5 \quad 0$

$\begin{array}{ll}0 & 5.9\end{array}$

$0 \quad 41.2$

$2.5(1.5) \quad 1.7(1.2)$

$1.9(0.6) \quad 1.5(0.6)$

$47.4(5.0) \quad 51.4(5.2)$

$1.9(0.4) \quad 1.7(0.5)$

$3.8(0.7) \quad 3.8(0.8)$

$*=p<0.05 ; * *=p<0.01, * * *=p<0.001$ 
Table 2: ToM in individuals with BPD, OPD, and in HC.

\begin{tabular}{|c|c|c|c|c|}
\hline & $\begin{array}{c}\text { BPD } \\
n=53 \\
\text { Mean (SD) }\end{array}$ & $\begin{array}{c}\text { OPD } \\
\mathrm{n}=34 \\
\text { Mean (SD) }\end{array}$ & $\begin{array}{c}\mathrm{HC} \\
\mathrm{n}=71 \\
\text { Mean (SD) }\end{array}$ & Statistics \\
\hline $\begin{array}{l}\text { MASC } \\
\text { Accurate ToM }\end{array}$ & $34.6(4.2)$ & $34.7(4.1)$ & $35.1(4.1)$ & $\begin{array}{l}\text { ANOVA: } F=0.28 \\
\text { BPD }=\text { OPD }=\mathrm{HC}\end{array}$ \\
\hline $\begin{array}{l}\text { MASC } \\
\text { Excessive ToM }\end{array}$ & $5.8(3.4)$ & $4.4(2.7)$ & $4.4(2.8)$ & Two-way mixed ANOVA. \\
\hline $\begin{array}{l}\text { MASC } \\
\text { Insufficient ToM }\end{array}$ & $3.3(2.1)$ & $3.9(2)$ & $3.9(2)$ & $\begin{array}{l}\text { Group: } F=0.31 \\
\text { Error: } F=77.55^{* * * *}\end{array}$ \\
\hline $\begin{array}{l}\text { MASC } \\
\text { No ToM }\end{array}$ & $1.4(1.5)$ & $2(1.6)$ & $1.6(1.4)$ & Error $\mathrm{x}$ group: $F=28.28 * *$ \\
\hline
\end{tabular}

$* *=p<0.01, * * *=p<0.001$

Table 3: Correlations between PD criteria and Excessive ToM error types

\section{Excessive ToM}

\section{PD criteria}

PD criteria other than BPD

Cluster A criteria

Cluster B criteria

Cluster C criteria

Borderline traits

$*=p<0.05, * *=p<0.01$

$$
\begin{aligned}
& \mathrm{r}=.29 * * \\
& \mathrm{r}=.29 * * \\
& \mathrm{r}=.24 * \\
& \mathrm{r}=.17 \\
& \mathrm{r}=.24 * \\
& \mathrm{r}=.18
\end{aligned}
$$


Figur 1: ToM error patterns in patients with BPD, OPD and HC

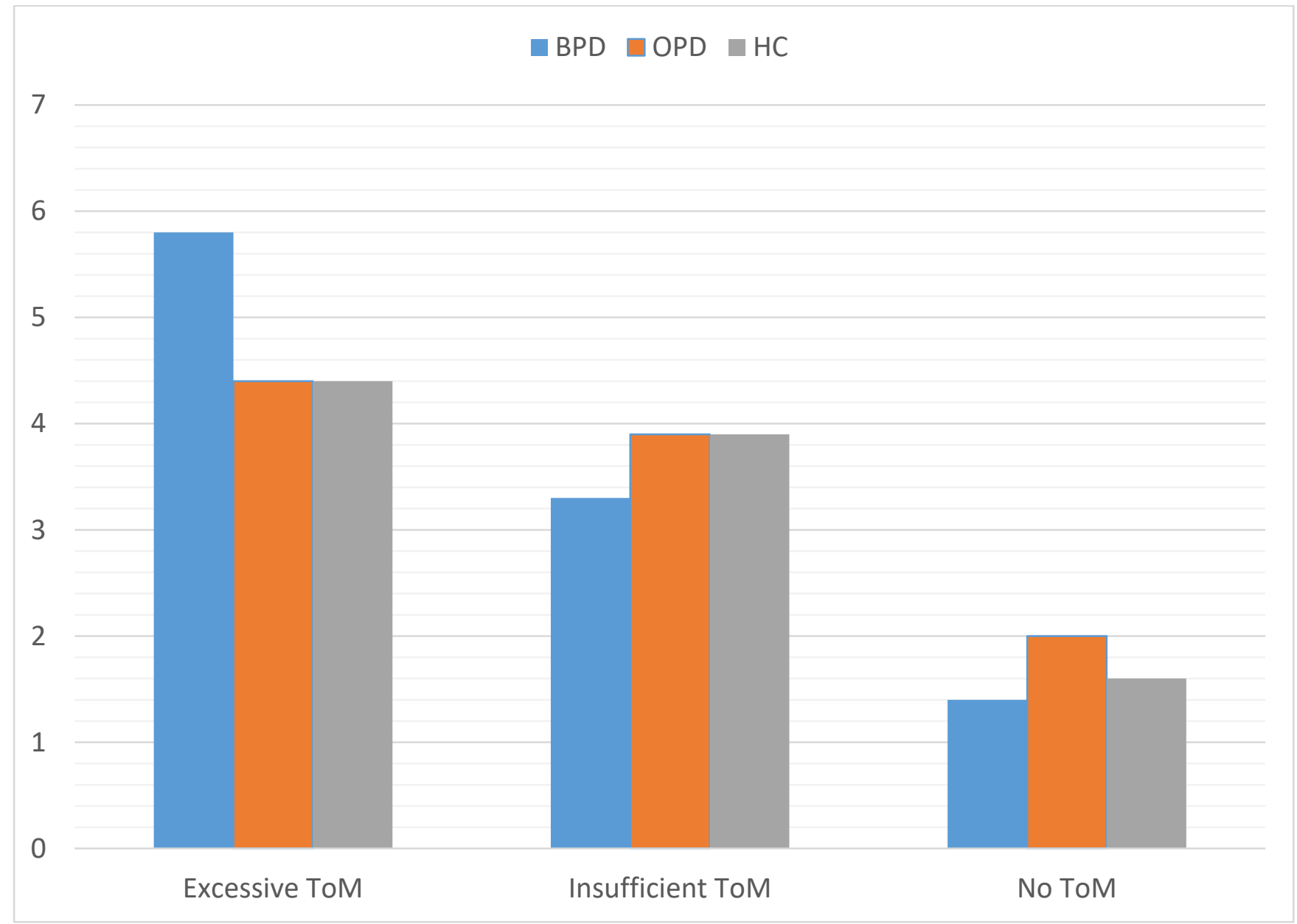

Excessive ToM: BPD > OPD* and $\mathrm{HC}^{*}$

$*=p<0.05$ 